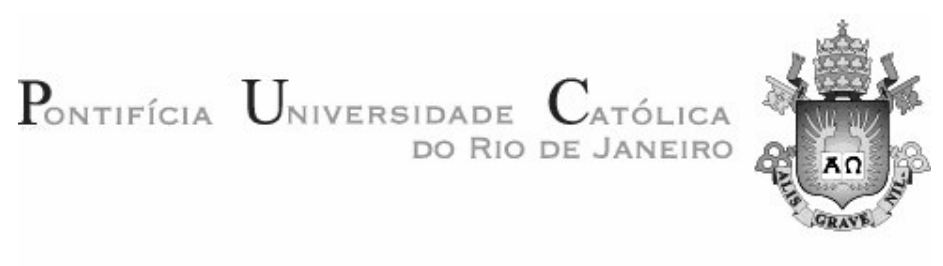

Flávia Barros Araujo

\title{
Como transformar informações em ações de marketing: um estudo de caso numa empresa de comércio eletrônico
}

\begin{abstract}
Dissertação de Mestrado (Opção profissional)
Dissertação apresentada como requisito parcial para obtenção do título de Mestre pelo Programa de Pós-Graduação em Administração da PUC-Rio.
\end{abstract}

Orientadora: Marie Agnes Chauvel 


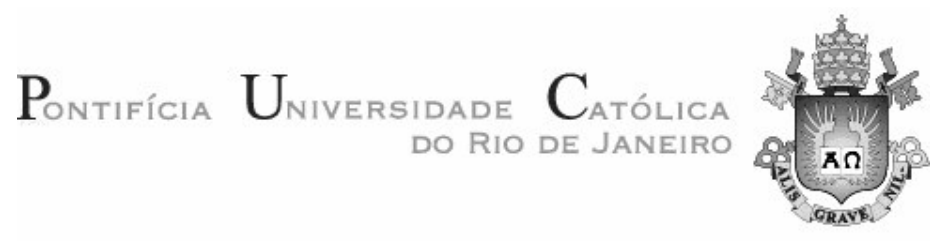

Flávia Barros Araujo

\title{
Como transformar informações em ações de marketing: um estudo de caso numa empresa de comércio eletrônico
}

\author{
Dissertação apresentada como requisito parcial \\ para obtenção do título de Mestre pelo Programa de \\ Pós-Graduação em Administração da PUC-Rio. \\ Aprovada pela Comissão Examinadora abaixo \\ assinada.
}

Prof. Dra. Marie Agnes Chauvel Orientadora

Departamento de Administração - PUC-Rio

Paulo César Motta Departamento de Administração - PUC-Rio

Letícia Casotti Departamento de Administração - COPPEAD/ UFRJ

Prof. João Pontes Nogueira Vice Decano de Pós-Graduação do Centro de Ciências Sociais - PUC-Rio 
Todos os direitos reservados. É proibida a reprodução total ou parcial do trabalho sem autorização da universidade, da autora e da orientadora.

\section{Flávia Barros Araujo}

É bacharel em Administração de Empresas pela Universidade do Estado do Rio de Janeiro (UERJ), graduando-se em 1997. Trabalhou na área de Inteligência de Marketing da Souza Cruz, foi trainee no Citibank em São Paulo e trabalha atualmente na B2W Companhia Global do Varejo. Seus interesses de pesquisa estão relacionados a Marketing de Relacionamento, técnicas de negociação e parcerias entre empresas.

Ficha Catalográfica

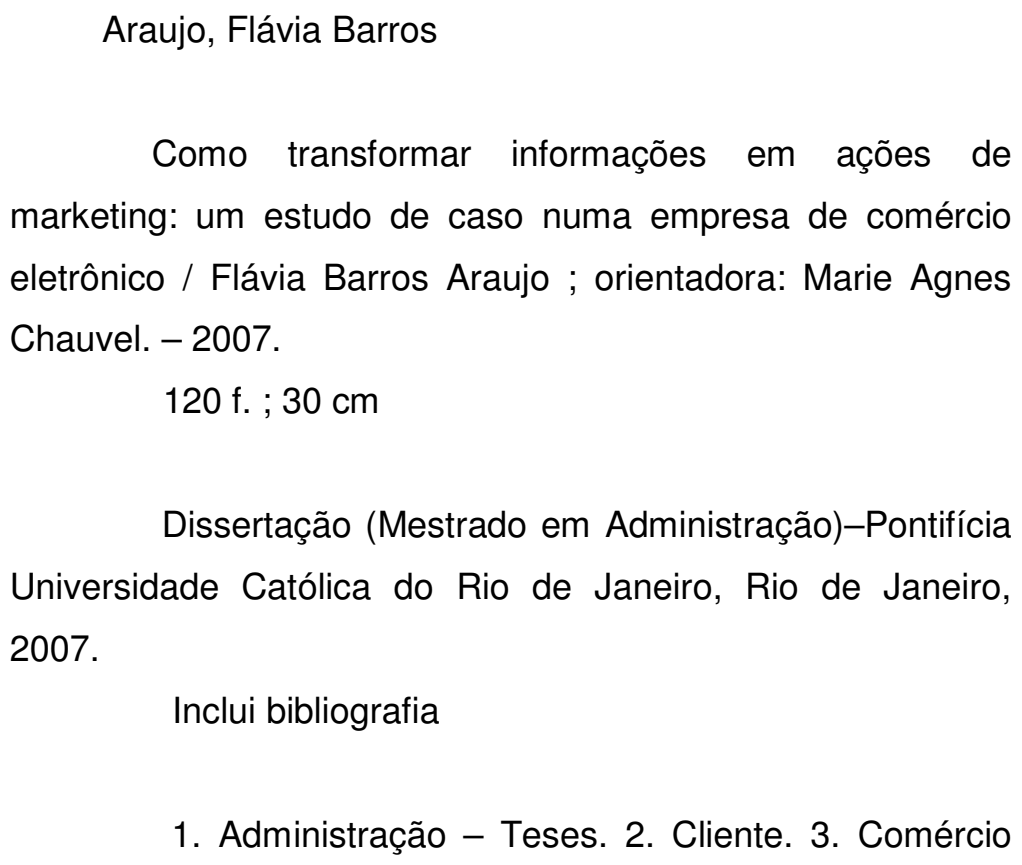




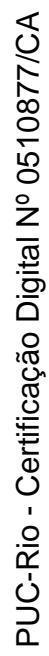

Para meus pais Mirian e Douglas, pelo amor, apoio e confiança. 


\section{Agradecimentos}

Agradeço a Deus pela oportunidade de fazer este curso e por me dar forças e saúde para conciliá-lo com meu trabalho.

Ao Vinicius Oppido de Castro pelo apoio, incentivo e companheirismo.

À minha orientadora Professora Dra. Marie Agnes Chauvel pela ajuda incontestável, pela compreensão frente às dificuldades enfrentadas e pelas sábias observações.

Ao Professor Dr. Paulo César Motta pelas aulas de Markerting de Relacionamento.

À Professora Hélene Bertrand pelas aulas de Comportamento do Consumidor.

Agradeço à PUC-Rio pela excelência dos professores e colaboradores.

Agradeço aos amigos Alexandre Canalini, Sergio Campos, Anderson Ribeiro, Vicente Rezende, Demetrius Ferreira, Ricardo Saito, Roberto Prudente, Renata Nunes, Rodrigo Goecks, Rosa Alcione Pinto Silva, Williams Campello, Rafael Laskier, Eduarda Berto Gouvêia, Joana Salgado, Bernardo Queima, Rafael Araújo, Rosilaine Barros, Tarika Lima, Berenice Oppido, Camile Scanferla, Claudia Mendes, Kátia Turon, Sully Turon, Paula Dutra, Thaiza Portella, Frederico Junqueira Jacobsen, Daniel Saldanha, Alda Marina.

A Teresa Campos e Fabio Etienne que muito ajudaram ao longo do curso. 


\section{Resumo}

Araujo, Flavia Barros; Chauvel, Marie Agnes. Como transformar informações em ações de marketing: um estudo de caso numa empresa de comércio eletrônico. Rio de Janeiro, 2007. 120p. Dissertação de Mestrado - Departamento de Administração, Pontifícia Universidade Católica do Rio de Janeiro.

Nos últimos cinco anos o comércio eletrônico brasileiro cresceu de forma significativa, fato que pode ser evidenciado através do aumento no número de pessoas fazendo compras pela Internet e do crescimento no volume de vendas pela Web. Paralelamente, a competição no varejo virtual tem se acirrado e a tarefa de conquistar e manter clientes se tornado mais árdua. $\mathrm{O}$ objetivo desse trabalho foi o de investigar como as informações provenientes de interações de clientes em sites de comércio eletrônico podem ser transformadas em subsídios para a tomada de decisões e em ações que auxiliem na gestão do relacionamento com o cliente. Para isso, foi feita uma revisão de literatura que abordou os temas comércio eletrônico e relacionamento com o cliente, com ênfase no uso do CRM (Customer Relationship Management) em varejo virtual. Em seguida, foi realizado um estudo de caso em uma das maiores lojas virtuais atuantes no país. O estudo teve por foco o processo de captura, armazenagem, transformação e utilização das informações de clientes dentro da organização, que foi descrito e analisado utilizando-se o quadro conceitual construído a partir da literatura. Os resultados apontaram que as informações de clientes da empresa analisada são utilizadas principalmente para planejar ações de promoção de vendas e para fins de segmentação na comunicação de ofertas. Foram identificadas algumas oportunidades para um uso de nível mais estratégico e voltado para o estreitamento da relação com os clientes.

\section{Palavras-chave}

Cliente, Comércio Eletrônico, Customer Relationship Management (CRM), Informação, Lojas Virtuais, Marketing de Relacionamento. 


\section{Abstract}

Araujo, Flavia Barros; Chauvel, Marie Agnes. How to convert information into marketing actions: a case study about an e-commerce company. Rio de Janeiro, 2007. 120p. Master's dissertation - Department of Business Administration, Pontifícia Universidade Católica Rio de Janeiro (PUC-RJ).

In the last 5 years the Brazilian e-commerce has grown significantly, that can be proved by the increase in the number of people who shops through Internet and the growth in the volume of web sales. Parallel, the competition at virtual market is tougher and the task of conquer and keep clients has become harder. The aim of this study was to investigate how information obtained from interactions of consumers in e-commerce web sites could be used to take decisions and actions to enhance the managing of the relationship with customers. For this, a literature review was done in subjects like e-commerce and relationship with customers, especially in CRM (Customer Relationship Management) in virtual market. After that, a case study was done about one of the biggest Brazilian companies of ecommerce. The study focused into the grab process, storage, analysis and handling of customer's information inside the company, which was described and analyzed using the literature's conceptual pattern. The results signalized that the company's customer information are used mainly to plan actions of sales promotion and to classify them for special offers. Some opportunities of usage in a more strategic level and aiming the tightening of this relationship with customers were identified.

\section{Keywords}

Customer, Electronic Commerce, Customer Relationship Management (CRM), Information, Online Shops, Relationship Marketing. 


\section{Sumário}

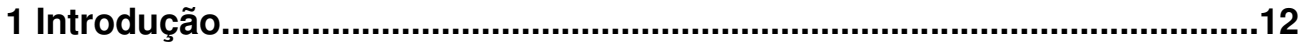

1.1. Contextualização do Problema.........................................................12

1.2. Formulação do Problema..................................................................14

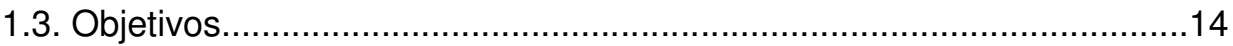

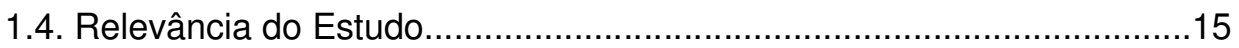

1.5. Delimitação do Estudo.................................................................17

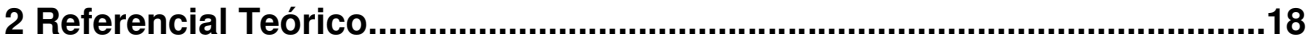

2.1. Comércio Eletrônico.....................................................................18

2.1.1. O conceito de comércio eletrônico e seu surgimento.....................18

2.1.2. O conceito de Loja Virtual........................................................24

2.1.3. Os Sites de Comércio Eletrônico no Brasil.....................................28

2.1.4. Síntese da Literatura sobre Comércio Eletrônico...........................31

2.2. O Relacionamento com o Cliente.....................................................3

2.2.1. A origem do foco no relacionamento com o cliente.......................33

2.2.2. Conceitos de Marketing de Relacionamento, CRM e

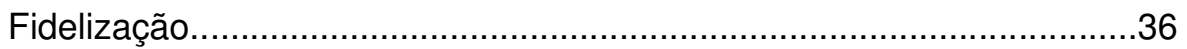

2.2.3. A importância de se investir no Relacionamento com o Cliente.....39

2.2.4. O processo de captura e tratamento das informações de

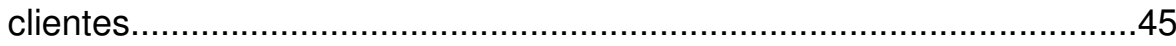

2.2.5. Ações de Marketing no contexto do Comércio Eletrônico...............57

2.2.6. Métricas para avaliação do desempenho de Projetos de CRM.....65

2.2.7. Panorama Atual do CRM..............................................................69

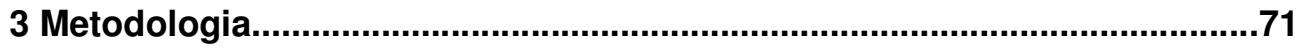

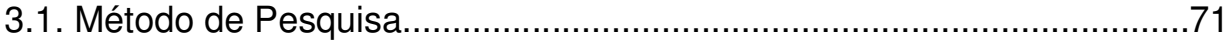

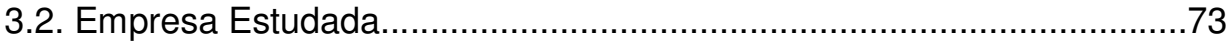

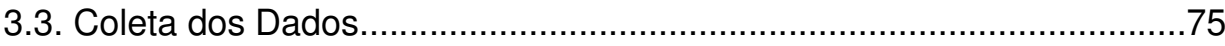

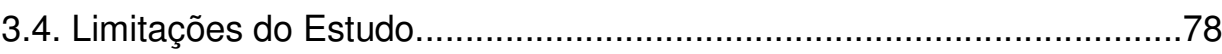


4 Estudo de Caso: $O$ caso da empresa Mercado Online S.A. ...................... 81

4.1. Tipos de dados de clientes capturados...............................................81

4.2. O processo de tratamento dos dados e os tipos de informações

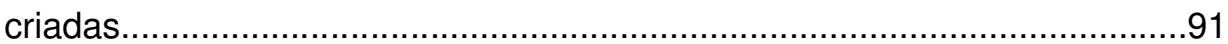

4.3. O uso das informações em tomadas de decisões.................................95

4.4. Tipos de ações criadas a partir de informações de clientes...................98

4.5. Lacunas identificadas no processo.....................................................101

4.6. Mensuração dos Resultados..........................................................105

5 Conclusões e Recomendações..................................................................107

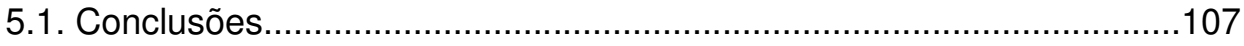

5.2. Recomendações para estudos futuros................................................112

6 Referências Bibliográficas......................................................................... 113

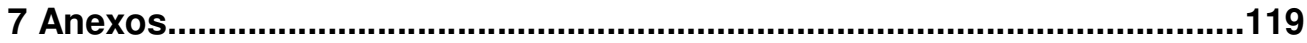




\section{Lista de figuras}

Figura 1: O ciclo do comércio eletrônico........................................................20

Figura 2: Como a Internet influencia a estrutura da indústria...........................22

Figura 3: Evolução do Faturamento do E-commerce no Brasil..........................28

Figura 4: Por que os clientes se tornam mais lucrativos com o tempo? ..............39

Figura 5: Curva de Clientes perdidos numa Companhia de Cartão de Crédito.. 40

Figura 6: A redução de $5 \%$ na taxa de clientes perdidos

Figura 7: Modelo de Cinco Etapas do Sistema de Conhecimento da Informação com o gerenciamento de necessidades para cada etapa.................46

Figura 8: A Estrutura do Modelo Empresarial para o CRM................................48

Figura 9: The Operations and Analytics Feedback Loop.........................49

Figura 10: Uma estrutura integrada de CRM................................................. 50

Figura 11: Construindo um DW centrado no cliente através do Modelo

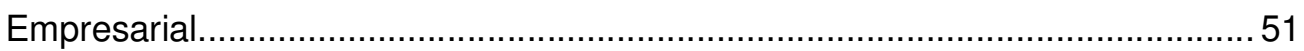

Figura 12: Seqüência de criação e entrega de valor ao cliente........................5 57

Figura 13: Exemplo de Portal na Internet e de espaços de mídia e venda..........82

Figura 14: Exemplo de resultado da busca do Google.....................................83

Figura 15: Pontos de Contato com o cliente.....................................................90 


\section{Lista de quadros}

Quadro 1: Participação das vendas por Categoria de Produtos.........................30

Quadro 2: Evidências, na Seguradora, de mudanças nas estratégias de gestão do relacionamento com o cliente........................................................43

Quadro 3: Método Antigo x Novo Paradigma..................................................54

Quadro 4: Quadro resumo do referencial teórico - tópico 2.2.4.........................55

Quadro 5: Capacidades do Comércio Eletrônico..............................................58

Quadro 6: Quadro resumo do referencial teórico - tópico 2.2.5......................... 64

Quadro 7: Quadro resumo do referencial teórico - tópico 2.2.6........................ 68

Quadro 8: Situações relevantes para diferentes estratégias de pesquisa...........72

Quadro 1: Informações capturadas nos pontos de contato com o cliente..........90

Quadro 10: Comparativo estudo de caso x referencial teórico do tópico 4.2......94 